\title{
Die Autoren
}

Dr Ino Arndt (†), Wissenschaftliche Mitarbeiterin des Instituts für Zeitgeschichte in Yünchen.

Dr. Heinz Boberach, Ltd. Archivdirektor im Bundesarchiv a. D.

Dr. Liliana Picciotto Fargion, Wissenschaftliche Mitarbeiterin am Centro di Docurentazione Ebraica Contemporanea in Mailand.

Dr. Hagen Fleischer, Professor für Neuere Europäische Geschichte an der Universitāt Kreta.

Dr. Frank Golczewski, Professor für Neuere Geschichte an der Universităt der Bundeswehr Hamburg.

Dr. Gerhard Grimm, Professor für osteuropäische Geschichte an der Universitāt München.

Dr. Gerhard Hirschfeld, Direktor der Bibliothek für Zeitgeschichte in Stuttgart

Dr. Hans-Joachim Hoppe, Lehrbeauftragter am Seminar für osteuropäische Geschichte der Universitāt zu Kōln.

Ostar Mendelsohn, Lektor in Oslo.

Dr. Jonny Moser, Vorstandsmitglied des Dokumentationsarchivs des österreichischen Widerstandes in Wien.

Dr. Gert Robel, Wissenschaftlicher Mitarbeiter am Osteuropa-Institut München und Professor an der Universitāt Innsbruck.

Dr. Eva Schmidt-Hartmann, Wissenschaftliche Mitarbeiterin am Collegium Carolinum in München.

Dr. Holm Sundhaussen, Professor für Südosteuropäische Geschichte am OsteuropaInstitut der Freien Univ. Berlin.

Dr. László Varga, Wissenschaftlicher Mitarbeiter am Institut für Geschichte der Ungarischen Akademie der Wissenschaften.

Hermann Weiß, Wissenschaftlicher Angestellter im Archiv des Instituts für Zeitgeschichte in München.

Dr. Juliane Wetzel, Wissenschaftliche Mitarbeiterin im Institut für Zeitgeschichte in München. Seit 1991 Mitarbeiterin am Zentrum für Antisemitismusforschung der TU Berlin.

Dr. Krista Zach, Wissenschaftliche Geschäftsführerin des Südostdeutschen Kulturverks in München. 
